\title{
An Analysis of the Influence of a Pseudo-haptic Cue on the Haptic Perception of Weight
}

\author{
Karljohan Lundin Palmerius, Daniel Johansson, Gunnar Höst and Konrad Schönborn
}

\section{Linköping University Post Print}

\section{Tweet}

N.B.: When citing this work, cite the original article.

Original Publication:

Karljohan Lundin Palmerius, Daniel Johansson, Gunnar Höst and Konrad Schönborn, An Analysis of the Influence of a Pseudo-haptic Cue on the Haptic Perception of Weight, 2014, Haptics: Neuroscience, Devices, Modeling, and Applications, vol 8618: 9th International Conference, EuroHaptics 2014, Versailles, France, June 24-26, 2014, Proceedings, Part I, Lecture Notes in Computer Science, 117-125

\section{http://dx.doi.org/10.1007/978-3-662-44193-0_16}

Copyright: 2014 Springer-Verlag Berlin Heidelberg

\section{http://link.springer.com/}

Postprint available at: Linköping University Electronic Press

http://urn.kb.se/resolve?urn=urn:nbn:se:liu:diva-109050 


\title{
An Analysis of the Influence of a Pseudo-haptic Cue on the Haptic Perception of Weight
}

\author{
Karljohan Lundin Palmerius, Daniel Johansson, \\ Gunnar Höst, and Konrad Schönborn \\ Department of Science and Technology, Linköping University, Sweden
}

\begin{abstract}
Haptics provides powerful cues about forces but cannot easily be integrated in all relevant applications, such as education. Pseudohaptic cues, visual information that simulate haptic sensations, have been raised as an alternative. It is, however, largely unknown how (or even if) pseudo-haptic cues are perceived by the haptic sensory modality. In this paper we present an approach that applies theories on multimodal integration to testing if a pseudo-haptic cue is triggering haptic perception. This approach is subsequently applied in designing an experiment that tests a pseudo-haptic cue based on a visual force-causes-displacement metaphor, similar to a rubber band.
\end{abstract}

Keywords: Pseudo-haptics, perception, cross-modal effects, multimodal integration

\section{Introduction}

The sense of touch has often proven useful in computer interaction, especially in applications where the conceptual understanding of forces is of importance. Examples include learning about electrostatic interactions or exploring scientific visualizations of flow data. Due to economical or technical reasons, however, haptic equipment is often inaccessible for direct application in these domains. In these cases pseudo-haptics could be an alternative. Pseudo-haptics is defined as the simulation of haptic sensations through visual stimuli in combination with sensorimotor actions [3]. Transferability of research with this focus can have potential beneficial cognitive implications for exploiting multimodal interfaces, such as supporting the learning of abstract science concepts.

While studies have shown potential benefits, the influence of pseudo-haptics on haptic perception is largely unknown. The current study aims to contribute to further work in this direction. The aim of this paper is twofold. Firstly, to devise an approach for testing the influence of pseudo-haptic cues on haptic perception. Secondly, to apply this approach in an experiment that investigates a common design of pseudo-haptics, based on a visual force-causes-displacement metaphor.

\section{Related Work}

In 2000 Lécuyer et al. presented a friction-causes-slow-down metaphor to represent friction force[4]. Since then, there have been numerous publications in 
the field, including studies on subjects' experiences with such feedback. For example, a pseudo-haptic feature of Richard et al.'s multimodal "Haptic Atomic" interface[9] allows a user to move an electron within a virtual atomic space to gain a sense of relative atomic energy levels. Paljic et al.[7] present a comparison between isometric and elastic pseudo-haptic cues for simulating torque. Their work provides indications as to what just-noticeable-differences (JND) to expect for the different cues, with possible implications for integration in a virtual environment. In addition, Rodgers et al.[10] present a pseudo-haptic approach for aiding users' handling of the transition between multiple screens in a multimonitor setup. Furthermore, Pusch et al. present a design based on a forcecauses-displacement metaphor in flow visualization[8]. For a detailed description of pseudo-haptic theory and a review of work on the topic see [3].

Biocca et al.[1] have explored the combined sensation of different cues in multimodal virtual environments. While primarily focused on measuring the level of presence in the environment, they also studied how subjects expressed their perception of "physical force" through a virtual spring pseudo-haptic metaphor.

Based on their previous findings, Lécuyer et al. have expressed the need to explore pseudo-haptic phenomena related to the weight of objects as well as the contrasts between force and pseudo-haptic feedback.

\section{Measuring the Haptic Influence of Pseudo-haptics}

In this study, we wish to measure the influence that pseudo-haptics has on haptic perception. To achieve this, we devised an experiment to measure the multimodal integration of pseudo-haptic feedback and real haptic feedback.

\subsection{Using Multimodal Integration as an Influence Measure}

Ernst et al. have shown (e.g. [2]) that when a physical attribute is presented by multiple sensory stimuli from different perceptual modalities, these stimuli will be combined into a single, more reliable, perception of the attribute. Such multimodal integration will occur even when the stimuli are somewhat conflicting. The degree to which each stimulus influences the integration is a function of an estimation of the quality of each stimulus. Two stimuli that are too conflicting or are perceived to represent different physical attributes will not be combined.

Based on the above, our idea for analysing cross-modal effects of pseudohaptics is as follows. Given that a visual pseudo-haptic cue is interpreted as a haptic attribute at a perceptual level, then that visual cue should be integrated with a haptic stimuli of the same attribute. By carefully adjusting the visual and haptic stimuli and measuring how the attribute is perceived, it should be possible to detect a cross-modal effect, if it exists. The physical attribute of weight is used in the present study. Adding visual cues to a haptic exploration of the weight of objects should allow for multimodal integration to take place. By adjusting the visual cues so that the weight of a heavy object is perceived to be more similar to the weight of a decoy object, the discrimination between the objects based on weight should deteriorate (see figure 1). 


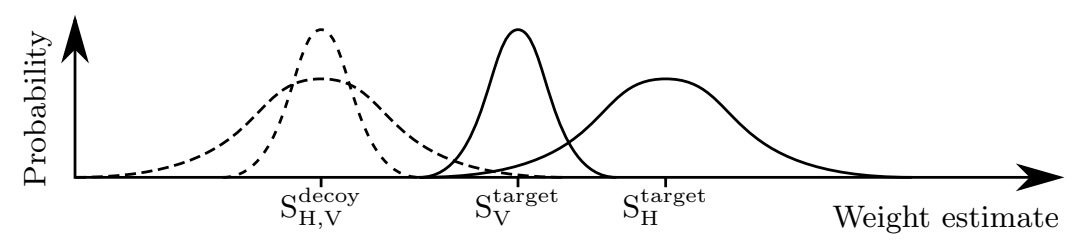

Fig. 1. Illustration of the probability functions of haptic $\left(\mathrm{S}_{\mathrm{H}}\right)$ and visual $\left(\mathrm{S}_{\mathrm{V}}\right)$ perception. The presence of multimodal integration between the target's haptic cues $\left(\mathrm{S}_{\mathrm{H}}^{\mathrm{target}}\right)$ and contradictory visual cues $\left(\mathrm{S}_{\mathrm{V}}^{\text {target }}\right)$ would theoretically shift the target weight estimate closer to the perception of the decoys $\left(\mathrm{S}_{\mathrm{H}, \mathrm{V}}^{\text {decoy }}\right)$, and thus worsen the JND.

\subsection{Study Design}

We designed a study to investigate the influence of pseudo-haptics on haptic perception of physical attributes through a single factor within-subject experimental design, using weight as the physical attribute. The experimental task was to select, among three virtual boxes, the box that was heavier than two identical decoy boxes that weighed less. The perception measure used as the dependent variable was the just-noticeable-difference (JND) in weight between the heavy box and the two decoys. The perceptual condition was used as the independent variable. Care was taken to design the study so that the integrated perception was measured and not only the visual and haptic modalities alone. Therefore, four different combinations of visual and haptic stimuli were used:

1. pure visual weight feedback, where a box is displaced downwards when lifted,

2. pure haptic weight feedback, where the haptic instrument applies real force,

3. haptic weight feedback and false uniform visual weight indication, and

4. haptic weight feedback and false contradictory visual weight indication.

Thus, the conditions were either purely visual, purely haptic or a combination of haptic and visual. Two variants of the combination of haptic and visual were used that differed in the visual cue. In Condition 3, the visual cue was uniform regardless of the weights of the boxes. In this condition the visual cue does not provide an indication of weight differences. In Condition 4, the visual cue actually contradicts the haptic cue by modulating the displacement to visually indicate that a heavier box is actually slightly lighter than the decoys. Although the outlined experiment measures haptic perception, the JND will be affected by multimodal integration and will therefore indicate any influence of the visual feedback on haptic perception.

\section{User Study}

The described study design was implemented in a user study. One challenge was the design of the visual pseudo-haptic feedback, which must provide an intuitive mapping between the visual cue and haptic interpretation to allow multimodal integration to occur. 


\subsection{Experimental Setup}

The design of the visual pseudo-haptic feedback was based on a force-causesdisplacement metaphor. This metaphor represents force by introducing a displacement of the visual representation of an object, when it is subjected to a force, similar to the visual appearance of lifting an object from a table using a rubber band. To emphasize the displacement, a line was displayed between the object and the point of attachment. This metaphor has previously been shown to make users explicitly associate the visual impression with the forces involved in the interaction.

The experimental setup was aligned with the three-alternative forced-choice approach (3-AFC). The 3D environment was designed to contain three boxes arranged horizontally (figure 2). Using the haptic device, users were able to grab the individual boxes and lift them to assess their weights. A selection marker was placed close to each of the boxes, and users could respond to the experimental task by pressing the marker associated to the box that they perceived to be the heaviest.

The experiment aimed to reveal subjects' subconscious haptic perceptions. To decrease the risk that subjects resorted to alternative visual strategies for assessing weight, frequent context switches were created by interleaving conditions. Furthermore, for each task, the period available for actively probing the weights of the boxes was limited to 20 seconds, from lifting the first box. After this time elapsed, the boxes disappeared from the screen, leaving only the selection markers.

Hypothesis Formulation The following hypotheses were formulated:

1. Pseudo-haptic cues perform better than purely haptic cues,

2. Purely haptic cues perform better than haptics with false visual cues, and

3. False visual cues perform better than haptics with contradictory visual cues.

Confirmation of the first hypothesis would indicate that our visual sense has a better JND than the haptic sense. Since this is known from earlier studies (e.g. [2]), we can use this as an indication that both the haptic and visual cues, and the experiment setup, worked as intended. Confirmation of the second and third hypotheses would indicate the presence of a cross-modal effect.

Stimuli Levels We deployed the psycho-physical staircase procedure [6], a technique for determining the intensity level that corresponds to a prescribed probability of response. A one-up-two-down sequence was used to determine the level at which the subjects had an accuracy $70.7 \%$. The step-size used was initially as large as $24 \mathrm{pp}$, so as to quickly converge to an approximate level, and then reduced to $0.5 \mathrm{pp}$ in two steps, as per an adaptive approach [5]. A pilot study was conducted to determine appropriate initial staircase and contradictory visual cue levels. The latter was set to $5 \%$. For further details on the staircase procedure see [6] for example. 
Based on the capabilities of the haptics hardware, the reference level was set to $0.1 \mathrm{~kg}$ with a gravity acceleration of $9.82 \mathrm{~m} / \mathrm{s}^{2}$. The amount of displacement in the pseudo-haptic cue was set to $40 \mathrm{~mm} / \mathrm{N}(25 \mathrm{~N} / \mathrm{m})$, to fit the interaction forces in combination with the size of the workspace.

Participants Fifteen ( $\mathrm{N}=15)$ subjects participated in the study, 5 females and 10 males (aged between 21 and 41). Data from one of the participants were removed due to a technical system error that prevented task completion, leaving 14 complete data sets. All participants were either familiar with haptic interaction or exposed to an introductory session prior to commencing the experiment. Participants received a complimentary meal as compensation for participating.

Data Collection Procedure Prior to the experiment, each participant was provided with identical instructions about how to perform the tasks. Subjects were free to take breaks between tasks when required. The experiment took approximately one hour to complete.

Each session commenced with a practice run that started with large stimuli levels $(50 \%)$, so that the participants could familiarize themselves with the environment and the four feedback combinations. Each feedback combination was presented in turn, with gradually reduced stimuli levels so that the subjects could learn how to interact and make decisions within the system when the tasks were also challenging for them. The subjects were instructed to explore the weight of the three displayed boxes and identify and select the heaviest box.

Following the practice run, participants were informed that the experiment commenced, and given the same task instruction. Throughout the experiment, the four conditions were tested in parallel. For each condition, the stimuli levels were determined according to the corresponding individual staircase. The experimental conditions were interleaved in a pre-determined repeating order, designed to provide subjects with a high frequency of context switches. For this reason, two alternative sequences of conditions were used: $1-3-2-4$ and $1-4-2-3$, respectively. Each sequence was presented to half of the subjects to minimize order effects. Since individual staircases were used, the number of iterations could differ between the conditions. However, to ensure that users still experienced context switches, tasks for at least one visual and one bi-modal condition were maintained until sufficient data was collected for all remaining conditions.

Post-experiment Questionnaire Upon completing the experimental session, each subject filled out a questionnaire in a debriefing discussion. The questionnaire consisted of four items where subjects rated, on a scale of 1 to 7,1 ) to what extent they used their sense of touch during the tasks, 2) to what extent they used vision, 3) to what extent they felt that their haptic and visual sensations were contradictory, and 4) to what extent they had a sensation of weight even when no haptic feedback was provided. A fifth free response item asked subjects to try to describe the sensations that they felt when they did not receive any 
haptic feedback. This questionnaire was designed to supplement the quantitative data by attaining subjects' conscious impressions of their experiences.

\subsection{Equipment}

The study was performed on a semi-immersive haptic workstation, see figure 2, running software based on the H3D API. This display system is equipped with a Desktop PHANToM device from Sensable, which is a high fidelity kinaesthetic haptics device based on the impedance control paradigm. The haptic feedback is co-located with stereoscopic graphics by reflecting an LCD monitor in a mirror over the haptic workspace. Users wear shutter glasses for stereo vision and the user interface is fully $3 \mathrm{D}$.
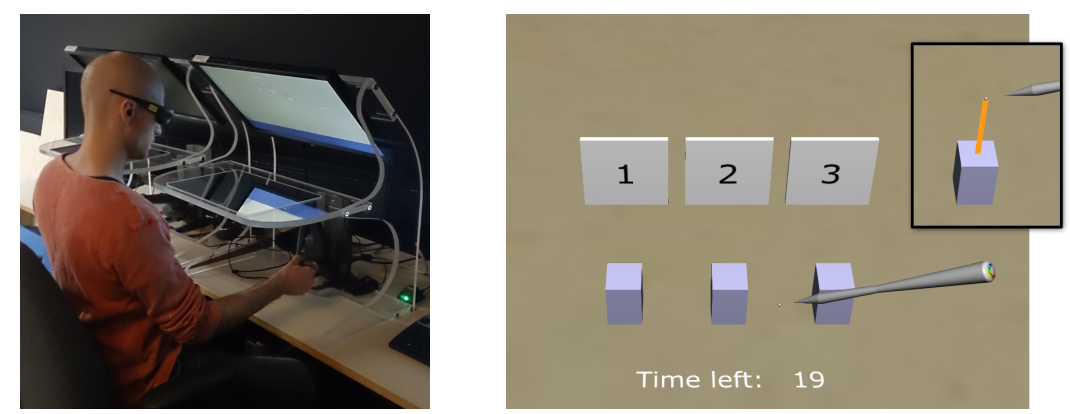

Fig. 2. The hardware (left) and a screenshot from the software (right) used in the experiment, with the pseudo-haptic cue shown in the inset image.

\subsection{Results}

For each participant, success in each experimental condition was estimated by calculating the JND based on the three last turning points in each staircase. This measure was designated as the dependent variable in the statistical analysis, using a decision criterion of $\mathrm{p}<0.05$.

Statistical analysis of the data obtained from the experiment $(\mathrm{N}=14)$ consisted of a one-way within-subject analysis of variance (ANOVA) of JND measures, using the experimental condition (1, 2, 3 or 4) as the independent variable. The ANOVA indicated a significant difference $(\mathrm{F}(3,39)=11.04, \mathrm{p}=0.00002)$ between the four conditions (table 1). Post hoc Bonferroni-corrected pairwise comparisons revealed that the participants were significantly more successful in the condition with pure visual weight feedback than in any of the other three conditions $\left(\mathrm{p}_{1-2}=0.00003, \mathrm{p}_{1-3}=0.015, \mathrm{p}_{1-4}=0.002\right)$. There were no other significant differences between the conditions.

The post-experiment questionnaire revealed that when asked to rate, on a scale of 1 to 7 , to what extent subjects felt that their haptic and visual sensations 
Table 1. Mean and standard deviations for the measured JNDs in each of the four experimental conditions.

\begin{tabular}{lcc} 
Condition & $\bar{x}$ & $\sigma$ \\
\hline 1. Pure visual & 0.0469 & 0.0320 \\
2. Pure haptic & 0.1308 & 0.0480 \\
3. Haptic with false visual & 0.1067 & 0.0548 \\
4. Haptic with contradictory visual & 0.1080 & 0.0429
\end{tabular}

Table 2. Collection of ratings provided by each subject $(\mathrm{N}=14)$ on the questionnaire.

\begin{tabular}{lc}
\multicolumn{1}{c}{ Question } & Subjects' ratings $(1-7)$ \\
\hline 1. Used touch during the tasks & 66557456676666 \\
2. Used vision during the tasks & 22431333112222 \\
3. Experienced contradiction & 43651323143516 \\
4. Experienced weight without haptic feedback & 64242232112221
\end{tabular}

were contradictory, two subjects revealed a rating of 6 , two of 5 , and two of 4 . Thus, six of the subjects felt that there was a degree of conflict when interpreting the bimodal sensory information. See table 2 for the full list of ratings.

Overall, subjects rated that they did not have a pseudo-haptic sensation of weight in tasks where no force feedback was present. A free response item asking subjects to try to describe in words the sensations that they felt when they did not receive any haptic feedback, however, revealed three interesting qualitative responses. These included, "A longer rubber band made the weight feel heavier", "The cubes with longer rubber bands felt slightly heavier", and, "The box felt heavier the longer the band was stretched".

\section{Conclusions}

We have presented an approach to test whether a pseudo-haptics design provides haptic perception, by considering the possibility of measuring cross-modal interference between visual pseudo-haptic and haptic cues. The experimental design was based on the principle that multiple stimuli representing the same physical attribute will be integrated into a single perception of that attribute. Specifically, we tested a pseudo-haptics cue based on a visual force-causes-displacement metaphor, analogous to stretching a rubber band.

Results showed that our pseudo-haptic cue resulted in a significantly better JND than the pure haptic cue (hypothesis 1 confirmed). This confirms previous findings in the field that vision has a better JND than touch. The absence of a statistical difference between pure haptic and multimodal cues (hypotheses 2 and 3 rejected) indicates that this particular design of pseudo-haptic feedback is, in our experimental setup, not perceived as representing the physical attribute of weight. The qualitative evaluation data suggested that at the conscious level at least, the received bi-modal sensory input was judged to invoke a degree of intermodal sensory conflict when solving the tasks. Furthermore, some participants 
revealed a haptic sensation in the language used to evaluate the experience of pure visual weight feedback.

In mirroring Lécuyer's contention[3] that science does not yet know with certainty whether any pseudo-haptic phenomenon is due to the design of the experiments aiming to measure it, or merely an associative perceptual mechanism, the results here also raise the question of what level of consciousness pseudohaptic phenomena operate at. Since the presented study investigates only one of many possible pseudo-haptics designs, a future study should consider the design of the pseudo-haptic cue, the physical attribute to be associated with the pseudo-haptic cue (e.g. magnetic instead of weight attributes), the sensorimotor actions that are associated with the pseudo-haptic cue, and further delineating the conscious and subconscious in the notion of pseudo-haptic sensations.

Acknowledgements This work is supported by the Swedish Research Council (VR) grant 2011-37694-88055-31. We thank Dr. Camilla Forsell for advice on the design of the study.

\section{References}

1. Biocca, F., Kim, J., Choi, Y.: Visual touch in virtual environments: an exploratory study of presence, multimodal interfaces, and cross-modal sensory illusions. Presence 10(3), 247-265 (2001)

2. Ernst, M.O.: A Bayesian View on Multimodal Cue Integration, chap. 6, pp. 105131. Oxford University Press (2006)

3. Lécuyer, A.: Simulating haptic feedback using vision: A survey of research and applications of pseudo-haptic feedback. Presence: Teleoperators and Virtual Environments $18(1), 39-53(2009)$

4. Lécuyer, A., Coquillart, S., Coiffet, P.: Simulating haptic information with haptic illusions in virtual environments. In: Procedings of the NATO RTA/Human Factors and Medicine Panel Workshop (2000)

5. Leek, M.R.: Adaptive procedures in psychophysical research. Perception \& Psychophysics 63(8), 1279-1292 (2001)

6. Levitt, H.: Transformed up-down methods in psychoacoustics. The Journal of the Acoustical Society of America 49(2), 467-477 (1971)

7. Paljic, A., Burkhardt, J.M., Coquillart, S.: Evaluation of pseudo-haptic feedback for simulating torque: a comparison between isometric and elastic input devices. In: Proceedings of the International Symposium on Haptic Interfaces for Virtual Environment and Teleoperator Systems (2004)

8. Pusch, A., Martin, O., Coquillart, S.: Hemp - hand-displacement-based pseudohaptics: A study of a force field application. In: Procedings of IEEE Symposium on 3D User Interfaces (2008)

9. Richard, E., Tijou, A., Richard, P., Ferrier, J.L.: Multi-modal virtual environments for education with haptic and olfactory feedback. Virtual Reality 10(3-4), 207-225 (2006)

10. Rodgers, M., Mandryk, R., Inkpen, K.: Smart sticky widgets: Pseudo-haptic enhancements for multi-monitor displays. Lecture Notes in Computer Science 4073, 194-205 (2006) 